\title{
LA COMPARACIÓN INTERTEXTUAL EN UNA APROXIMACIÓN AL TEXTO TRADUCIDO DENTRO DE LA TRADUCTOLOGÍA DESCRIPTIVA
}

Jesús M. SANCHEZ GARCIA

Universidad de Córdoba

o.

En este artículo nos proponemos hacer una serie de consideraciones sobre el papel que desempeña y que debe desempeñar la comparación entre un texto (TO: texto origen) y su traducción (TM: texto meta) dentro de la llamada Traductología Descriptiva (TD). El trabajo se enmarca en nuestra investigación sobre los desplazamientos léxico-semánticos pertinentes al tema del amor en la traducción del Cuarteto de Alejandría de L. Durrell, sobre la que ya hemos ofrecido diversos aspectos (véanse nuestras referencias en la bibliografía final). Muy resumidamente, consiste en la confección de un corpus léxico (fundamentado en el estudio del componente lexicológico desde el enfoque de $\mathbf{M}$. Mingorance ${ }^{1}$ ), que nos facilita la localización asistida por ordenador de los entornos topológicos del texto que corresponden a una lectura o interpretación cognitivamente representativa de la relación traductiva entre la novela original

1 Enfoque sobre el que se basan los importantes trabajos de Felices (1991), y Faber y Mairal (1994, 1995a y 1995b). Véanse en la bibliografia final, asimismo, las referencias más significativas de $\mathbf{M}$. Mingorance. 
y su traducción española. De esta manera obtenemos un corpus transémico que se analiza según una versión ampliada de Van Leuven-Zwart (1989-90) y que comentamos interpretativamente atendiendo a criterios que diseñamos basándonos en la teoría del texto.

I.

Un estudio de la praxis de traducción en un momento dado y en un caso determinado ayuda enormemente a delimitar el grado de consolidación o cambio del sistema literario meta (receptor) que es producido por la traducción literaria, o más bien por su resultado, es decir, por la literatura refractada ${ }^{2}$. En este sentido nuestra investigación proporciona una modesta base para otros estudios «de seguimiento» con un corte más sociológico y literario.

La investigación global en la que se inserta este artículo versa sobre el aspecto semántico-textual de la manipulación implícita en dicha refracción, como base que pudiera servir para delimitar a posteriori, en otro tipo de estudio, el alcance ideológico-cultural de la refracción del traductor (e incluso, naturalmente, del traductólogo, esto es, de nuestro trabajo descriptivo). La lingüística puede perfectamente explicar los aspectos que se prestan a tratamiento lingüístico, que no son pocos, aunque lógicamente no pueda agotarlos.

En nuestra opinión, este tipo de comparación de un solo texto traducido con su original debe ser la base imprescindible sobre la que realizar el comentario crítico de los textos traducidos y sacar conclusiones empíricamente fundamentadas sobre lo que implica globalmente lo que llamamos traducción literaria.

La comparación lingüístico-textual entre TO y TM, tal y como la exponemos en nuestro estudio general ${ }^{3}$, es valiosa para determinar las constricciones que se dan en la producción de una literatura en el sistema meta y las estrategias de este sistema literario-cultural en la selección o rechazo del material procedente de otra cultura. La comparación intertextual traductológica cumple así un cometido muy importante dentro del área de la literatura comparada, el de investigar cómo afectan a la producción de una traducción literaria tales limitaciones - resultado en última instancia de la ideología cultural- mediante decisiones lingüístico-literarias.

2 Lefevère (1981 y 1984b) presenta una imagen del traductólogo literario como agente que refracta las refracciones (manipulación del significado textual cribado mediante una poética y una ideología).

${ }^{3}$ Cfr. p. e. Sánchez, 1993 y 1995 a. 
Dentro de la TD se han avanzado algunas propuestas de metodología descriptiva con las que nuestro enfoque coincide parcialmente. La que propone Toury (1980) consiste básicamente en identificación de unidades de traducción (textémica), establecimiento de la «traducción adecuada» (TA), comparación textémica con sus elementos correspondientes en el TM y descripción de las relaciones traductivas; sin embargo, no incluye ninguna clasificación de los textemas y se hace difícil la descripción sistemática de desplazamientos. Tampoco dice qué criterios emplear para determinar cuáles son los elementos correspondientes. Uno de sus epígonos (Broeck, 1985) retoma esta metodología, y aboga por una declaración sobre la norma del traductor, previa al análisis textémico, que establecería el grado de equivalencia en los diversos niveles (fónico, léxico, etc.) y su comparación con la TA. ${ }^{4}$

Otro enfoque que parte de una comparación exhaustiva y un análisis de los efectos de los desplazamientos en las categorías literarias ${ }^{3}$ para averiguar sus motivos y efectos es la metodología transferencial de Frank. Como nosotros, Frank aboga también por segmentar el texto narrativo aunque lo hace según las categorías literarias sobre la novela y recurre a la crítica literaria y la poética del autor para guiar la selección de pasajes pertinentes. A este traductólogo de la Universidad de Göttingen, que defiende un enfoque heurístico, textual e histórico-cultural ( Ubersetzungskultur») le parece saludable empezar con preguntas como

what concepts and methods are most promising for this particular research problem, in this particular case, which is part of such and such corpus involving such and such literature, language, and cultural pair, and located at that particular moment in time? (Frank 1989:88).

Este tipo de cuestiones nos sirvio también para plantear nuestra investigación, enmarcarla en una metodología ${ }^{6}$ en la que hemos procurado flexibilizar los conceptos linguísticos y pensar en su aplicación heurística y concreta al caso concreto que nos ocupaba.

4 Resulta difícil pensar cómo establecer las normas si no es a partir de los desplazamientos en las relaciones textuales. De hecho así se implicaba ya en Toury (1978:93), ya que se considera la comparación TO-TM como la parte más importante de los estudios comparativos (que también incluirian la comparación de varias traducciones de un mismo original y la comparación de aspectos del TM con aspectos similares de otras obras en la lengua del TM); sin esta comparación intertextual, que justifica el estudio de una sola traducción de un solo original, no se podría determinar la «norma inicial * ni siquiera en términos de adecuación.

Suponemos que se incluiría aquí la categoría tópico o tema.

- Cfr. Sánchez (1995a). 
Newmark propone una crítica traductiva histórica dentro de la literatura comparada que cubra: el análisis del TO, averiguando su intención y sus aspectos funcionales - por qué es así- así como su relación con el léxico y el tema; la interpretación y el método del traductor; comparación detallada y selectiva de TO y TM, especialmente con relación a la interpretación del tema de la obra; evaluación; finalmente valoración del lugar que ocupa la traducción en la cultura meta. Nuestro método tiene que ver con los tres primeros pasos.

Lambert y Gorp presentan un modelo en el que se parte de unos datos hipotéticos preliminares, que incluyen la metaliteratura (crfíca interpretativa) sobre la obra en cuestión, para preparar el análisis posterior macro y microestructural. En el primero dividen el texto en tipos de narrativa (diálogo, descripción, monólogo, etc.), tratan la estructura narrativa interna, etc. En el segundo, incluyen los cambios o desplazamientos ${ }^{7}$ léxico-semánticos, junto con otros de otro tipo, aunque no dicen cómo tratarlos, y confrontan este análisis con el macroestructural.

La hermenéutica de Steiner incluye un modelo histórico y otro psicologico del lenguaje para la teoría de la traducción, que tendría así esta doble vertiente. En nuestra opinión, la traductología descriptiva se ocupa de descripciones concretas sometidas a cambios y revisiones históricos, aunque se requiera un enfoque textual previo ; y es también psicológica en el sentido de que, aun partiendo de la semántica léxica, como en nuestro caso, necesita la complementación que supone el enfoque cognitivo, tanto en el eje paradigmático como en el sintagmático. En cualquier caso, el movimiento hermenéutico («hermeneutic motion») necesita del análisis textual del TO y de algún modelo comparativo que establezca semejanzas y diferencias entre los dos textos.

El hecho de segmentar el texto con propósitos descriptivos, procedimiento del que somos partidarios, no debe hacernos olvidar que la segmentación se hace en virtud de la estructura conceptual por la que el intérprete se representa el texto, correspondiendo la segmentación al área de la estructura conceptual más pertinente para el análisis textual. No se puede decir todo sobre lo que se dice en el texto, parece advertirnos Birch (1989:21), pero se pue-

7 Preferimos «desplazamientos», y sus implicaciones cognitivas, para verter «shift* al espahol, recogiendo la concepción de Neubert (1985:8) del cambio traductivo como «displaced communication». Esta dimensión tampoco es recogida por el concepto similar de trajection propuesto por Malone (1988: 9) dentro de la lingüística aplicada a la traducción.

- Todo estudio traductologico concreto es un acto histórico de interpretación, ya que «the translator can only commit himself to a demonstration of how he considers the text to be relevant in given historical and local circumstances» (Broeck 1986:108). 
de mejorar mucho, sin embargo, si intentamos explicar nuestra propia idea (interpretación) construida ex profeso acerca del texto.

La segmentación, aunque realizada con criterios conceptuales, debe producir «manageable chunks» (Toury, 1986) que funcionen en la comparación textual como unidades de traducción ${ }^{9}$, o mejor, como configuraciones de unidades de traducción para la comparación y al diseñarlos no se debe caer en procedimientos demasiado arbitrarios, peligro muy frecuente, ya que una porción o segmento textual es un fragmento variable que solo puede determinarse en cada caso particular (Lörscher 1989:65).

Nuestro corpus transémico ${ }^{10}$ representa no un texto sino un conjunto delimitado de fenómenos de traducción extraído de un corpus textual, las novelas del Cuarteto. Es en este conjunto fenoménico donde acontecen los desplazamientos ${ }^{11}$. Su análisis es el único instrumento de que disponemos para entender el proceso de traducción.

La tarea de la traductología descriptiva no es lejana a la crítica traductológica propuesta por Newmark (1981:182). Esta tendría por cometido ayudar a la interpretación de la obra de autores y traductores concretos, y su procedimiento incluye el análisis temático, el léxico de autor y el establecimiento de comparaciones sobre la base de la diferencia entre TO y TM «including in particular their interpretations of subject-matter». Este esbozo erradica la falsa idea de que hay descripción traductológica sin ejercicio crítico, o crítica exclusivamente evaluativa; la evaluación sólo es una dimensión de esta crítica que plantea Newmark (ibid.) y la labor descriptiva cae de lleno en ella.

Coincidimos plenamente con Voort cuando dice que las traducciones son siempre interpretaciones macroestructurales y que los fenómenos propios de la meta-interpretación ${ }^{12}$ del analista-traductólogo (desplazamientos incluidos) deben definirse siempre con respecto a una visión particular del texto. Para nosotros esta visión la compone la lingüística textual con base léxico-semántica y dimensión cognitiva, que nos parece desemboca naturalmente en una crítica textual cooperativa. Consideramos muy importante su idea de que la

- Se ha sefralado que aunque se suele operar con la oración como unidad básica de la actividad traductora, el centro de esta radica mayormente en las unidades léxicas del texto (Newmark 1991:66).

10 Cfr. Sánchez (1993, 1995a).

1 Véase más adelante.

12 Como se ve, Voort coincide totalmente con nuestra concepción del traductólogo como intérprete en segunda instancia que refocaliza la focalización previa que realiza el traductor a traves de los desplazamientos, entendidos éstos como ejemplos de interpretacion traductiva. 
interpretación, sería en realidad una «memorial synthesis» ${ }^{13}$ (1991:68) construida tanto por el traductor como por el traductólogo gracias al menos en parte a las cadenas léxicas cohesivas (a partir de los elementos microestructurales). Los macrodesplazamientos se producen en virtud de dicha síntesis, la cual lo es también de los elementos contextuales y extratextuales pertinentes, como es la poética implícita o explícita del autor. Esto justificaría, en nuestra opinión, realizar una crítica de la novela o tener en cuenta la crítica existente de un determinado autor para la traducción o para el análisis de la traducción.

Consideramos que en toda traducción se da una interpretación crítica ${ }^{14}$ procedente de unas decisiones microestructurales que responden a lo que Toury llama norma operativa. Koster distingue entre esta interpretación y la interpretación crítico-literaria ${ }^{15}$ : la interpretación traductiva es «not the view as it develops itself during the process of translation but the specific view on the source text as it emerges from the translation product» (17). Sin embargo, pasa por alto que este emerger desde el producto es ya un nuevo acto interpretativo - de las relaciones TO-TM-y que por tanto la interpretación traductiva depende necesariamente de nuestra concepción del TO y del TM tal y como surge en nuestro proceso de lectura de ambos textos por separado y siempre teniendo en cuenta que tienen, para los propósitos del traductólogo, un carácter relacional. Más adelante se verá el alcance de esta precisión ${ }^{16}$. Lo importante para él, como para nosotros, es averiguar cómo describir las posibilidades interpretativas del TO y ver cuáles se plasman en el TM, así como qué cambios o desplazamientos microestructurales hay y qué macroefectos

13 Es de lamentar que no indique más explícitamente por qué derroteros debe transcurrir la confección de esta síntesis. Aquí las contribuciones de la lingǘrstica textual ya exploradas- y el enfoque cognitivo son inestimables.

14 La posición del traductor como cierta especie de critico y la reproducción de este hecho en el caso del traductólogo -crítico de un crítico- ha sido muy bien vista por Broeck (1985:61). La relación entre traductologia y crítica es la culpable del estancamiento en la disciplina que observa Lefevere (1980:15), y que habría surgido según Lambert (1991:30) de la distinción que hacen Holmes y Toury entre ambas, reservando la identificación sólo en el caso de la Traductología Descriptiva histórica. Welt (1980:37), sin embargo, sugiere que el traductor es un crítico involutario, lo quiera o no; nosotros así lo entendemos, estando más de acuerdo con Bakker (1990) cuyo método obliga al analista a explicitar todo proceso de actualización o semantización de un semema (es decir, su selección a expensas de otros) y considera la descripción misma como un interpretante, igual que la propia traducción, pero en una nueva etapa de semiosis o producción de significado. (Es clara aquif la herencia de Peirce).

is Sin embargo, hay escuelas, como la eslovaca, que si vinculan directamente los estudios de traducción con la crítica literaria (cfr. Hochel 1991:41).

16 Pero nótese que según ella resulta diffcil no asignarle a los procesos interpretativos mencionados un estatuto en última instancia crítico-literario. 
producen. Creemos que un enfoque como el nuestro, basado en una aplicación lexicológica/lexicográfica a la descripción traductológica, tiene mucho que decir en este sentido.

Proponemos que el estudio comparativo debe basarse primeramente en los mecanismos cohesivos en tanto que pistas comunicativas orientativas para el traductor, al constituir una sólida base (isotópica) para la interpretación textual en el plano semántico-intensional ${ }^{17}$. A nuestro juicio, la tarea consistiría en parte en intentar explicar cómo se alcanza una representación mental dada en el TM y ver cómo difiere de la alcanzada para el TO. Al mismo tiempo se debe explorar el efecto de una palabra particular del TM sobre su coherencia global en relación al TO. En esta operación radicaría la «translatorische Relativitat» (Neubert, 1986) de la que para nosotros se desprende que en el comentario de las fórmulas de un pasaje del TM no se pueden obviar las consecuencias para todo el contexto, ya que las diferencias en las elecciones léxicas llevan a condiciones cotextuales diferentes; las configuraciones léxico-semánticas, que contribuyen al significado macrotextual gracias a las redes de coherencia que emanan de los significados léxicos, no serían similares para ambos textos ${ }^{18}$. Vemos entonces cómo los cambios o desplazamientos traductivos serían entonces el resultado de una interpretación traductiva inherente al proceso traductivo.

II.

Las operaciones traductivas, como resultado de las preferencias más o menos ideológicas del traductor, movilizan en el potencial lingǘstico parámetros semántico-pragmáticos no carentes de una dimensión conceptual que pueden utilizarse como tertium comparationis en la comparación de las estructuras actualizadas de dos textos en dos lenguas diferentes. Desde nuestro punto de vista la comparación es la verdadera razón de ser de la traducción y de la traductología y no por ello se debe pensar como Bassnett y Lefevere (1990:4) que concebir una base de comparación es necesariamente caer en juicios de valor sobre la calidad de una traducción. Supone interpretación, que no evaluación necesariamente, y, como la interpretación del traductor, implica decisiones (en el mejor de los casos intersubjetivas) sobre la importancia que da el analistaintérprete al material lingüístico.

17 Cfr. Albaladejo (1992).

tema del original, y en que se debe prestar minuciosa atención al realce de las cadenas de imágenes. 
El significado de las opciones léxico-semánticas se puede relacionar con su interpretación recurriendo al concepto linguístico-textual de tópico o tema. En función de su pertenencia a un proyecto interpretativo global, podemos decir que aquellas son pertinentes (que exhiben, para un intérprete dado, la facultad de estar relacionadas).

Toda comparación produce pares traductivos cuyos rasgos prototípicos incluirían, según Cruse (1992:19), el grado de semejanza semántica entre los textos, el grado de semejanza estructural de los textos, el grado de informatividad proposicional de los textos, el grado de disparidad entre los sistemas linguísticos respectivos y el grado de formación correcta de los textos. Nuestra propuesta metodológica, centrada en el texto desde el sistema, abarca todos estos criterios excepto los dos últimos, aunque el tercero viene en parte explicado por la estructuración contrastiva del campo del amor ${ }^{19}$.

El enfoque variacional en traductología descriptiva (Hewson y Martin, 1991) se basa en la comparación entre opciones traductivas productoras de significado más que en el estudio de la conversión de unas fómulas en otras. El nombre «variacional» deriva de la utilidad que los autores ven en estudiar las condiciones linguísticas virtuales de variación y las variaciones concretas disponibles para el traductor condicionadas por la CM (cultura meta).

El principio fundamental que para ellos gobierna el proceso de producción del metatexto y la comparación es la homología, es decir la aceptación de la diferencia como fundamental y el estudio de las semejanzas sobre esa base. También aceptan, como nosotros, y a diferencia de otras tendencias, el principio de direccionalidad ineludible en toda traducción, ya que el TM permanece siempre en una relación significativa con el TO como resultado de la aceptación de unas opciones en detrimento y exclusión de otras.

Su orientación es hacia la generación de traducciones más o menos válidas más que hacia una descripción de los fenómenos observados comparando opciones ya dadas o hechos de traducción consumados. Debemos concluir que su enfoque, con toda la validez que podemos observar en él, nos parecería mucho más adecuado si se englobara en la rama aplicada de la disciplina, aunque proporciona un marco metodológico muy interesante para la pedagogía de la traducción.

El procedimiento indica el estudio de relaciones interproposicionales analógicas como cadenas de secuencias cohesivas en la (re)construcción del texto, teniendo en cuenta que en toda construcción textual se redefinen las elecciones semánticas del plano proposicional.

${ }^{19}$ Cfr. Sánchez (1995b). 
Parece justificarse entonces nuestra ponderación de las elecciones léxicas mediante el análisis textual del TO, conducta un tanto denostada por parte de algunas escuelas traductológicas. Al mismo tiempo se favorece, en este enfoque como en el nuestro, la inserción de los metatextos o fragmentos textuales previos (p.e. críticos) que son pertinentes en lo que los autores denominan conexión texto-intertexto, producida por la lectura personal del lector o traductor. Ello justifica que el análisis transémico deba realizarse con la inclusión de la información que se considera pertinente, además del comentario de dicho análisis.

La aplicación natural de esta representación homológica generativa a la pura descripción de las relaciones traductivas existentes de facto la podríamos realizar en teoría contemplando la gama de interpretaciones que somos capaces de producir y considerando las opciones expresadas en el TM como soluciones con un doble sistema de relaciones variacionales: (a) hacia el TO; (b) hacia el resto de opciones pertenecientes a la gama variacional del TM. En traductología estrictamente descriptiva se suele considerar únicamente (a), y así lo haremos nosotros, para no incurrir en la crítica evaluativa que entrañaría más o menos solapadamente (b), la cual se considera más propia de la rama aplicada.

En toda traducción hay cambios, y se ha señalado con frecuencia que todo proceso de reescritura produce, por ejemplo, una mayor especificidad o generalidad en el sentido ${ }^{20}$ textual, que nosotros atribuimos más que nada a los cambios en el significado léxico. Es de destacar aquí la obra de Popovic, quien en los albores de la disciplina acepta que son inherentes a la traducción. Estos desplazamientos propios de la traducción acaban modificando las relaciones textuales. Estamos plenamente de acuerdo con Gentzler (1993:103) cuando pone de relieve la necesidad de que se llegue a un consenso sobre los métodos descriptivos sistemáticos, como paso previo para poder producir estudios de las traducciones de un autor o un traductor, de un período, género, lengua o cultura, o incluso de historias generales de traducción. Nuestra investigación de la traducción de la novela de L. Durrell pretende apuntar en esta dirección.

Abundando en esta línea, Blum-Kulka (1986:23) reconoce el efecto que tienen los desplazamientos sobre la cohesión y coherencia textuales, hablando de «desplazamientos de cohesión» ${ }^{21}$, los cuales alterarían las funciones

20 No entendemos como desplazamiento, y por tanto no lo recogemos así para el corpus transémico, si interpretamos el mismo sentido textual para dos textemas con designaciones aparentemente divergentes, ya que, como afirma Coseriu (1977:229), el traductor se ve obligado a veces, para mantener el sentido textual, a elegir una opción léxica que supone un cambio de designación en relación al TO.

${ }^{21}$ (Greimas 1976:246) ya explicaba cómo los cambios
lístico (por ejemplo, cohesivo) y la estructura sémica total. 
producidas por las redes cohesivas léxicas, así como de «desplazamientos de coherencia», para los que defiende la necesidad de un estudio psicolingǘrstico. Nos parece que el problema estribaría sobre todo en determinar, a partir de la cohesión, la centralidad de las alusiones pertinentes al desarrollo de la acción y del tema. En el análisis habría que centrarse en los desplazamientos de índole textual (ya que estos son más evitables que los originados por y en el lector), concretamente en los que ella llama opcionales, es decir, no motivados directamente por la diferencia existente entre los sistemas lingüísticos.

K. van Leuven-Zwart (1989-90) elabora un método de descripción y comparación de traducciones, del que nos servimos para la parte traductológica de nuestra investigación. Aunque, a diferencia de ella, nosotros proponemos una metodología con pretensiones de representatividad en la obtención de un corpus transémico homogéneo sobre el que efectuar los análisis, el suyo es un método muy útil y un excelente punto de partida y nosotros lo retomamos con ciertas modificaciones para el estudio de los desplazamientos en la traducción española del Cuarteto, entre otros motivos porque se basa en la intersubjetividad de los significados léxicos y de las respuestas a la hora de establecer relaciones y conexiones entre fenómenos dispares.

Los conceptos fundamentales que dan pie a la metodología son el de relación de semejanza y el de disparidad entre categorías, que suelen producirse casi siempre simultáneamente. Los desplazamientos resultantes afectan todos a la función interpersonal y por tanto a diversos aspectos del mundo de ficción, y son producto las más de las veces de la interpretación del traductor. Asimismo, forman un esquema que exhibe regularidades ${ }^{22}$. Como hemos visto ya, estamos plenamente de acuerdo en que estas se deben analizar a través de la comparación y la descripción de una sola traducción, si se quiere enriquecer la investigación teórica de la traductología, ya que de no hacerlo, como bien dice van Leuven-Zwart, la orientación puede dejar de centrarse en la traducción como producto para convertirse en objeto de estudio de disciplinas relacionadas pero ajenas.

La norma traductiva que es de especial interés para nosotros es la operativa (Toury, 1978), la cual regula decisiones durante la traducción y es de dos tipos: matriciales, que regulan la segmentación textual, y textuales, que determinan las pertinencias que se establecen (Rabadán 1989:402). Parece necesario entonces examinar en primer lugar las modificaciones que tienen lugar y que afectan a segmentos textuales, para después reconstruir el método interpretativo probable, en la interpretación del traductólogo, que ha seguido

$22 \mathrm{O}$, diríamos nosotros, tendencias al menos. 
el traductor en su activación de pertinencias. Si el traductor activa conocimientos pertinentes, realiza operaciones manipuladoras sobre el texto, éstas no son ajenas a las operaciones del traductólogo.

Aunque partimos de que los desplazamientos son inevitables, surge el problema de distinguir hasta que punto éstos influyen en la interpretación del analista o si, por el contrario, el concepto que se tiene de interpretación es crucial a la hora de establecer los desplazamientos.

Claramente la relación es dialéctica e influye en la formulación de hipótesis sobre la interpretación del traductor, y las normas y la estrategia de traducción; no podemos describir traducciones sin interpretar ambos textos y viceversa.

Preferimos pensar que cuando leemos o traducimos, interpretamos macroestructuralmente tras hacer acopio de microinterpretaciones que van siendo afianzadas en función de la tesis que uno va adquiriendo hasta que al final ya se tiene una macrointerpretación global (que no total, pues siempre habrá relecturas). No obstante, se puede argüir que la descripción de arriba a abajo se sustenta en el hecho constatado de que primero se lee el original y luego se determinan los desplazamientos comparando paso a paso. Nosotros hemos preferido otorgar a la primera hipótesis macroestructural un estatuto intuitivo apriorístico que queremos apoyar en el micronivel semántico, el cual nos conduce a una interpretación más afianzada de la macroestructura.

Nuestro enfoque defiende la representatividad de los fragmentos textuales, sobre los que realizar la comparación y la interpretación de los desplazamientos del corpus, y los criterios textuales para su obtención, a diferencia de van Leuven-Zwart, que propone estudiar las partes del comienzo de los capítulos de la obra, sin razón aparente que lo justifique.

Para nuestros pares léxicos de comparación en que postulamos la existencia de un architransema, con van Leuven-Zwart, retenemos la concepción de Van den Broeck según la cual se puede considerar el TO y el TM como dos ejemplares del mismo tipo, el cual vendría a representar el núcleo invariante de una relación de suyo disimilar, al menos en parte. El concepto de equivalencia es entonces conceptual ${ }^{23}$ y semiótico-textual, considerando el autor la teoría textual y semiótica como parte de la teoría de la traducción. Esta es también la línea sugerida por Nida, quien propone un enfoque sociosemiótico de la traducción centrado en la equivalencia funcional, es decir, de funciones, ya que las lenguas difieren más en la manera de comunicar que en

${ }^{23}$ Cfr. Newmark (1988: 162), donde señala que la llamada «cognitive translation» correspondería al núcleo invariante. 
lo que comunican. No por afirmar esto, creemos, se estaría abrigando planteamientos radicalmente universalistas. Sin embargo, es cierto que un concepto siquiera aproximativo de equivalencia es necesario y útil, si se quiere explicar, por ejemplo, por qué, cuando leemos a Dostoievsky, a pesar de hacerlo en retraducción via francés o inglés, entendemos tantos aspectos de los temas que trata. Proponemos que el núcleo invariante es de orden conceptual pero con implicaciones discursivas claras: se deben abordar las dimensiones semiótico-textuales y cognitivas del tertium comparationis entre unidades textuales del TO y del TM.

El objetivo de van Leuven-Zwart, fiel a su línea product-oriented, es establecer y describir los desplazamientos en la traducción de textos literarios narrativos, para lo que dispone de dos submodelos, el modelo comparativo, que clasifica los microdesplazamientos por el nivel lingüístico afectado dentro de las oraciones, cláusulas o sintagmas, y el modelo descriptivo propiamente dicho, que establece el efecto de los microdesplazamientos sobre la macroestructura, lo que podríamos llamar también macroefectos. El método sería también la base de una traductología enfocada al proceso de traducción ya que desde él se puede indagar en las opciones del traductor en tanto que correlatos de sus objetivos textuales más inmediatos.

Sostenemos que no se puede discutir la función de un TM en la CM sin hacer un análisis textual del TM, que indefectiblemente aparece en el nuevo polisistema literario o sociocultural en algún tipo de relación con otro texto cronológicamente anterior; esta direccionalidad es parte de la ontología de la traducción, que debe aceptarse más que criticarse, aunque se tenga más interés en un polo que en otro, como ocurre con Toury, cuyo interés por el sistema meta nos parece injustificadamente excesivo.

Los desplazamientos que van Leuven clasifica incluyen los semánticos, estilísticos y pragmáticos ${ }^{24}$. Para la comparación se manejan una serie de conceptos: transema es una «comprehensible textual unit» (155) basada en los marcos predicativos de la Gramática Funcional (GF) de S. C. Dik de modo que

24 Dicha clasificación, a nuestro entender, debe perfeccionarse más aún y recibir una mayor fundamentación lingúístico-teórica, terreno este que nos parece muy prometedor para investigaciones futuras. Cfr. Olbertz (1987) para una interesante y juiciosa restructuración de algunas subcategorias del modelo comparativo de van Leuven-Zwart (relativas a las categorias madificación sintáctico-semántica y modulación y modificación semánticas) desde una reconsideración de la función y asignación de Sujeto, y del concepto de perspectiva, dentro del marco de la Gramática funcional de Dik. Por su parte Broeck (1978:43) considera, como nosotros, que el componente clave del estilo es el semántico, lo cual hace necesario una interpretación funcional del significado. aunque, a diferencia de nuestra propuesta -que nos parece más explícita-, no se nos dice cómo se especifica el concepto de función. 
tendríamos transema de estado de cosas ${ }^{25}$ (verbo léxico y argumentos) y transema satélite con algunas de las características de los satélites de Dik. Este último transema funciona como una especificación adverbial o ampliación del primero. Sin embargo, pensamos que sería más acertado proponer textema en vez de transema, sirviéndonos de Toury, ya que van Leuven-Zwart no distingue entre transema del TO o del TM como unidad textual, por un lado, y las relaciones de semejanza y contraste entre las unidades de ambos textos (que son las que sirven en primer lugar como criterio interpretativo para la segmentación), por otro. Reservamos, por lo tanto, el término transema para esta relación en tanto que ineludible fenómeno cognitivo de la traducción que se manifiesta entre textemas que se pueden disponer en corpus transémicos para el análisis y utilizamos textema en lugar de transema. Obsérvese que el «translema» de J. C. Santoyo consiste en kuna unidad bitextual de cualquier tipo o nivel constituida por un mismo contenido y dos manifestaciones formales diferenciadas pero solidarias y cuya existencia depende de la relación global de equivalencia» (Rabadán 1989:409). No hemos utilizado «translema», sin embargo, por encontrarse este término íntimamente ligado a un concepto de equivalencia no del todo semejante a nuestra concepción de la relación transémica, en otras palabras, por ser demasiado theory-laden. Sí estamos de acuerdo, sin embargo, en que la relación de traducción debe concebirse dinámicamente -admitiendo cierta parcela de indeterminación-y como una postulación única para cada caso particular de descripción traductológica (cfr. Rabadán 1989:409).

En cuanto a atextema» nos parece muy útil su introducción por hallarse ya en Toury y porque se ha reiterado numerosas veces su importancia. Así, EvenZohar (1979:629) considera el textema como la unidad literaria por excelencia (ya que en la traducción literaria se revela el sistema de relaciones textuales y literarias que encierra una obra de arte verbal). No debe extrañar que la identificación de textemas se considere cuestión problemática. Si hay dos análisis (basados en última instancia en una intuición sobre la macroestructura), habrá dos series textémicas y dos relaciones traductivas diferentes. Esto es así porque las relaciones transémicas no se encuentran en los textos for the taking sino que se construyen en el análisis. Las nuevas relaciones paradigmáticas y sintagmáticas del TM ponen en juego la codificación del textema en el código e insertan en el

25 Newmark (1992:50) ha señalado que el enunciado oracional sigue ocupando un lugar destacado como segmento/unidad de traducción, por lo que no es casualidad que van Leuven-Zwart haya escogido el marco predicativo de Dik para su segmentación. Ivir (1981:217) se ha expresado en parecidos términos sobre las decisiones procedimentales según el valor apreciado en cada «unidad comunicativaw y en su entorno. 
nuevo sistema literario nuevas funciones de los signos que se manifiestan en forma de tensión en cada segmento entre aquéllos y sus funciones. La identificación del textema, que es en sí misma un acto interpretativo, nos ofrecería así un modo económico de estudiar las relaciones entre ciertos signos y sus signos cotextuales, aunque la descodificación textémica plantee todavía muchos problemas. Creemos que nuestra investigación arroja cierta luz sobre sus posibles soluciones.

Nuestro transema representa pues una pareja problema-solución establecida a partir del TO y el TM e inserta en relaciones textuales pertinentes. Según Toury (1980b:87), estas relaciones se organizan jerárquicamente, y con las de otras parejas se forma la jerarquía de relaciones pertinente para todo el texto. $\mathrm{Pa}$ ra nosotros las jerarquías son estructuradas en el proceso cognitivo de lectura de cada texto; una vez lé́dos los textos y en el proceso de comparación, se forman las jerarquías transémicas, que dan cuenta del binomio microestructura/macroestructura para el fenómeno traductivo que une los dos textos a la vez.

Otro concepto en el método de van Leuven-Zwart es el de relación, procedente de la semántica estructural, en el que la semejanza es condición de la desigualdad u oposición. El architransema (ATR) es otro concepto importante que hace referencia al denominador común o núcleo invariante expresado en metalenguaje no gramatical (sólo sustantivos, verbos, adjetivos y adverbios). El concepto de invariante es espinoso, ya que ciertos autores no conceden crédito alguno a una teoría de la invariante, aunque admiten el componente diferencial. Aún así pensamos que no se puede prescindir del comparatismo en cualquier juicio descriptivo o interpretación (sea lingǘstica o literaria), y sf se puede reunir una serie de principios sobre cómo describir dicha invariante, sin los que es muy difícil hablar de pérdida o déficit semántico, parámetro que, por otra parte, todos los autores reconocen. Para la identificación del ATR Van Leuven-Zwart aconseja utilizar criterios propios de una tradición intersubjetiva como la lexicografi$\mathrm{ca}^{26}$ (158). En realidad el ATR está basado, aunque no se dice explícitamente, en

26 La traductologia comparte muchos criterios con la lexicografia, como el de necesitar reivindicar validez intersubjetiva para las interpretaciones de sus análisis, aunque en los análisis del traductólogo sólo interviene el criterio de una sola persona. De ahí que apoyemos el análisis transémico del corpus en la teorfa lexicológicalexicográfica desarrollada desde amplias perspectivas que se mueven siempre animadas por un espíritu cooperativo que busca el consenso intersubjetivo. En cuanto a la relación entre cohesión y coherencia, fundamental en el análisis textual y transémico, de Petofi (1984) se desprende que semejante metodología es crucial para establecer las relaciones interlexémicas sobre las que realizar la interpretación, y resalta la importancia de estructurar dichas relaciones según los métodos de los diccionarios monolingües si se quiere aplicar las representaciones léxicas a la semántica textual. Estas representaciones podrian limitarse a los elementos léxicos que más influyen en la interpretación textual y transémica. 
la noción de archilexema de Coseriu y otros autores procedentes de la teoría de los campos léxicos, al igual que la noción de norma acuñada por el lingüista rumano fue importada a la traductología sobre todo por Toury. Es obvia la pertinencia de aplicar el método de van Leuven-Zwart para describir el corpus transémico a una metodología como la de $\mathbf{M}$. Mingorance, que proporciona a su vez los cimientos para la obtención de nuestro corpus léxico ${ }^{27}$. Consideramos ambas metodologías las piedras angulares de nuestro trabajo, con las debidas transformaciones a las que las hemos sometido.

Una mejora, creemos, que se debe introducir en los aspectos de conjunción y de disyunción descritos por Van Leuven-Zwart, es la indicación del campo semántico en la descripción transémica del corpus, como lo es también el uso de la descomposición léxica de los elementos de los textemas que sufren variación ${ }^{28}$. Tomamos así, en primera instancia, el significado de cada uno de estos elementos léxicos como si fuera la entrada léxica de un lexicón como el representado en la metodología de $\mathbf{M}$. Mingorance, utilizando su mismo método para las definiciones. Después de todo, el valor de análisis por componentes es alto «si uno ve la traducción como un reordenamiento disciplinado de componentes de significado comunes a dos comunidades linguísticas» (Newmark 1992:162,191) ${ }^{29}$.

Los desplazamientos más importantes se producen como sigue: hay modulación cuando hay sinonimia entre un textema y el architransema, e hiponimia entre el otro y el ATR. Si ambas relaciones son hiponímicas con el ATR, obtendremos una modificación y si no se puede establecer ATR alguno, habrá una mutación. En realidad nosotros creemos que la frontera entre ausencia de desplazamiento y mutación es borrosa y sería mejor pensar en estas categorías como prototipos, de manera que los ATR representaran casos centrales o periféricos de la conjunción o disyunción entre textema y architransema. En cuanto a la hiponimia, ésta se cifra en la fórmula $X$ es un tipo, una clase, un modo de $Y .^{30}$ Los desplazamientos más comunes suelen ser las modulaciones semánticas especificativas y la modificación sintáctico-semántica explicativa de palabras estructurales. Ambos son atribuibles al comprensible intento de facilitar el acceso al TO.

Según los estudios de la macroestructura textual ${ }^{31}$ las unidades significativas de la macroestructura narrativa, (como la acción, el punto de vista, los

27 Cfr. Sánchez (1995b).

23 Cfr. Sánchez (1995a) para una ejemplificación de como quedaría el análisis de los transemas.

${ }^{29}$ Naturalmente nosotros no sólo la vemos así, aunque este es un aspecto básico de nuestra concepción y metodología.

30 Remitimos a Leuven-Zwart (1989) para una ejemplificación de los tipos de desplazamientos y para una discusión general más detallada.

${ }_{31}$ p.e. Dijk (1977 y 1990). 
episodios con sus acontecimientos, etc.) dependen de características microestructurales. Van Leuven-Zwart toma de Bal (1990) y de Leech y Short (1981) - quienes a su vez se basan en las metafunciones de Halliday (p.e. 1985) - la justificación teórica para unificar las funciones del lenguaje con los niveles del discurso y de la fábula intensional o story. Desde la perspectiva de la función ideacional, el primero, ofrecería para el estudio las elecciones semánticas que producen el estilo cognitivo (mind style) y el segundo el mundo narrativo como objeto de focalización. Nuestro interés principal versa sobre los microdesplazamientos semánticos cuando éstos se producen sobre un nodo (es decir, un miembro del campo léxico-conceptual que nos interese estudiar -como el del campo del amor ${ }^{32}$ - con función cohesiva). Creemos que es aquí donde se deben centrar los estudios de la traducción del tema narrativo. Beaugrande (1980:41) llama la atención sobre las mutaciones que afectan a estos nodos, porque el trastorno de la cohesión que ello implica transforma el mundo textual tal y como lo percibimos cognitivamente. Toca entonces establecer la forma en que los microdesplazamientos traen como consecuencia macrodesplazamientos en el estilo cognitivo del amor y cómo éstos pueden traducirse en desplazamientos en el marco del amor de la macroestructura. En consecuencia nos encontramos ante una situación en la que la microestructura semántica contribuye a la macroestructura ideacional del discurso. Cada una de las elecciones lexicosemánticas contribuye a la expresión del significado temático interproposicional mediante la construcción de relaciones cohesivas y por el hecho de que forman relaciones paradigmáticas dentro del espacio conceptual. La cohesión así formada nos proporciona el vínculo que en un propósito similar al nuestro se necesita establecer entre los campos léxicos y la estructura narrativa para aplicarlo al estudio de la traducción. Desde un punto de vista teórico, dicho vínculo tiene lugar en la zona de transición entre las microestructuras semánticas y la macroestructura ideacional del discurso, es decir, en un espacio conceptual dinámico que explica en nuestra opinión gran parte de la borrosa indeterminación identificada tantas veces en la interpretación del significado de un texto.

Partiendo de que las secuencias proposicionales que satisfacen el tópico textual funcionan como marcos cognitivos con su propia macroestructura jerárquica según niveles de información, en la discusión del corpus que debe hacerse en un estudio de este tipo se deben comentar las relaciones que vinculan los marcos activados a acontecimientos narrativos (en los cuales se pueden producir desplazamientos semánticos), por lo que resulta importante averiguar qué estructuras

32 Cfr. Sánchez (1995a y 1995b). 
conceptuales se movilizan en relación a los architransemas que regulan los desplazamientos. Los desplazamientos que nos interesan, por tanto, acontecen en las porciones textuales correspondientes a las fases dominantes del mundo narrativo ya que corresponden a los medios textuales más pertinentes.

Por su parte, la descripción de los macrodesplazamientos supone un paso del discurso a la fábula y por tanto un paso interpretativo que hay que determinar. Cada desplazamiento tiene la capacidad potencial de causar determinados macroefectos ${ }^{33}$. Es difícil determinar qué grado de consistencia o regularidad es necesario para que se dé un macroefecto propiamente dicho, y esta es una decisión, en última instancia, interpretativa.

Los cambios en el estilo cognitivo pueden producir desplazamientos en la caracterización de los personajes o en las emociones o acciones. Según van Leuven-Zwart, si hay un desplazamiento en la función ideacional del discurso, se producirá uno paralelo en la función ideacional de la fábula; por ello, resulta útil concebir estos dos niveles narrativos semiunidos, y concentrarnos en ellos en la descripción que nos interesa. Dichos cambios ideacionales pueden mermar el conjunto de historias paralelas (señaladas por Eco, 1987) que son producidas como resultado de las múltiples interpretaciones ofrecidas potencialmente por el texto,

Como posibles resultados, y en principio, es conveniente pensar que se pueden dar desde muchos microdesplazamientos con ligeras desviaciones hasta pocos desplazamientos con un macroefecto considerable.

Finalmente, y teniendo presente lo que recomienda la autora holandesa sobre el particular, en la discusión o comentario del corpus transémico se deberían expresar también el número de desplazamientos por transema, su naturaleza, categorías y tipo, otras tendencias, tipo de interpretación (coherente/sistemático $v s$. serial o extemporáneo), y estrategia y norma traductivas.

\section{Biblografia}

Albaladejo MAYORDOMO, T. (1992). «Aspectos pragmáticos y semánticos de la traducción del texto literario», Koiné, II (1-2), 179-200.

BaKkER, MATTHuS (1990). «The Task of the Describer: Between Two Meanings of "Interpréte", Series of Leuven pre prints, conferencia presentada en la " 2 nd Cera Chair for Translation Studies» de Lovaina, Agosto, 1990.

33 Van Leuven-Zwart describe los macroefectos probables sobre el discurso y sobra la fábula intensional. 
BAL, M. (1990). Teoría de la narrativa, Madrid: Cátedra.

BASSNETT, S. y LeFEVERE, A. (ed.) (1990). Translation, History and Culture, Londres y N.Y.: Pinter.

BEAugrande, R. de (1980). «Towards a Semiotic Theory of Literary Translating», en W. Wilss (ed.) Semiotik und Úbersetzen, Tübingen: G. Narr, 23-42.

BIRCH, DAVID, (1989). «Shifts of Cohesion and Coherence in Translation», en J. House y Shoshana Blum-kulka (eds.) (1986), Interlingual and Intercultural Communication, Tübingen: G. Narr, 19-35.

BRoECK, R. van den (1978). «The Concept of Equivalence in Translation Theory: Some Critical Reflections», en J. S. Holmes et al. (eds.) (1978), Literature and Translation: New Perspectives in Literary Studies, Leuven: Acco, 29-68.

BROECK, R. van den (1985). «Second Thoughts on Translation Criticism: A Model of Its Analystic Function», en Hermans, T. (ed.), The Manipulation of Literature, Londres: Croom Helm, 54-62.

BroECK, R. van den (1986a). «Generic Shifts in Translated Literary Texts», New Comparison: A Journal of Comparative and General Literary Studies, 1, 104116.

COSERIU, E. (1977). «Lo erróneo y lo acertado en la teoría de la traduccion» en $E l$ hombre y su lenguaje. Madrid: Gredos, 214-239.

CRUSE, D. (1992). «Levels of Translation: A Linguistic Perspective» en M. Thelen y B. Lewandowska-Tomaszczyk (1992), 19-34.

Duk, T.A. van, (1977). «Semantic Macrostructures and Knowledge Frames in Discourse Comprehension» en M.A. Just y P.A. Carpenter, (1977), 3-32.

Duk, T.A. van (1983; 1978). La Ciencia del Texto: un enfoque interdisciplinario, Barcelona: Paidós.

Duk, T.A. van (1990). «Issues in Functional Discourse Analysis», en Pinkster, Harm y Genee, Inge (eds.) (1990), Unity in Diversity, Dordrecht: Foris, 27-46.

Eco, U. (1987; 1981). Lector in Fabula. La cooperación interpretativa en el texto narrativo, (trad. esp., Ricardo Pochtar) Lumen: Barcelona.

EvEn-ZoHaR, I. (1979): «The Textemic Status of Signs in a Literary Test and its Translation», en Chatman, S., Eco, U. et. al. (eds.), A Semiotic Landscape: Proceedings of the first congress of the IASS, Milan, June 1974, The Hague: Mouton, 629-633.

FABER, P. y MAIRAL, R. (1994), «Methodological Underpinnings for the Construction of a Functional Lexicological Model», Miscelánea. A Journal o English and American Studies, 15, 193-217.

FABER, P. y MAIRAL, R. (1995a). «The Paradigmatic and the Syntagmatic Structure of the Semantic Field of Existence in the Elaboration of a Semantic Macronet», Studies in Language, (en prensa).

FABER, P. y MAIRAL, R. (1995b). «Semantic Frames and Dimensions. Towards a unified approach», International Journal of Lexicography' (en prensa). 
FELICES, A. (1991). El componente axiológico en el lenguaje. Su configuración en los adjetivos que expresan emociones y conducta en la lengua inglesa. Tesis doctoral: Universidad de Granada.

FRANK, A. P. (1989). «Translation as System» and Übersetzungskultur: On Histories and Systems in the Study of Literary Translation», Comentarios introductorios efectuados en el Symposium *History and System in the Study of Literary Translationw, organizados por el Göttingen Centre (Sonderforschungsbereich «Die Literarische Ubersetzung») en Abril, 1989.

Frank, A.P. (1986). «Towards a Cultural History of Literary Translation», REAL, Berlín/New York: De Gruyer, 317-380.

GeNTZLER, EDWIN (1993). Contemporary translation Theories, NY. y Londres: Routledge.

Greimas, A.J. (ed.) (1976). Ensayos de semiótica poética, Barcelona: Planeta.

Halliday, M.A.K. (1985). An Introduction to Functional Grammar. Londres: Edward Arnold.

Hochel, B. (1991). «The Cross-Temporal and Cross-Spatial Factors and the Translation of Literary Language» en $K$. van Leuven-Zwart y T. Naaijkens (eds.) Translation Studies: The State of the Art, Proceedings of the First James S. Holmes Symposium on Translation Studies, Amsterdam: Rodopi, 41-48.

Holmes, J.S. (1970). The Nature of Translation, The Hage: Mouton.

IVIR, V. (1981). *The Communicative Model of Translation in Relation to Contrastive Analysis», en W. Kühlwein y W. Wilss München: W. Fink, 209-218.

KOSTER, C. (1990). «The Concept of Interpretation in Descriptive Translation Studies: First Explorations», Series of Leuven pre prints, conferencia presentada Second $\mathrm{Ce}$ ra Chair for Translation, Communication and Cultures, Agosto, 1990, Lovaina.

KOSTER, C. (1991). «How Do We Observe the Kind of facts Translations Are?», Series of Leuven pre prints, conferencia presentada 3rd Cera Chair for Translation, Communication and Cultures, 18 de julio de 1991 en Lovaina.

LAMBERT, J. y GORP, H. VAN (1985). «On Describing Translations», en T. Hermans (ed.) The Manipulation of Literature: Studies in Literary Translation, Londres: Croom Helm, 42-53.

LAMBERT, J. (1991). «Shifts, Oppositions and Goals in Translation Studies: Towards a Genealogy of Concepts» en K. van Leuven-Zwart y T. Naaijkens (eds.) Translation Studies: The State of the Art, Proceedings of the First James S. Holmes Symposium on Translation Studies, Amsterdam: Rodopi, 25-37.

LEECH, G. (1981). Style in Fiction. A Linguistic Introduction to English Fictional Prose. Londres y N.Y.: Longman.

LEFEVERE, A. (1980). «The Relevance of the German Tradition», Pacific Quarterly Moana 5 (1) (núm. esp. de traducción), 5-16.

Lefevere, A. (1981). «Translated Literature: Towards an Integrated Theory» The Bulletin of the Midwest Modern Language Association, 14 (1), 68-78. 
LEFEVERE, A. (1984a). «Translations and Other Ways in Which One Literature Refracts Anothern, Symposium: A Quarterly Journal in Modern Foreign Literatures, 38 (2), 127-142.

LEFEVERE, A. (1984b). That Structure in the Dialect of Men Interpreted», en Shaffer, E.S. (ed.) Comparative Criticism, vol. 6, CUP, 87-100.

Leuven-Zwart, K. Van (1985). *The Methodology of Translation Description and Its Relevance for the Practice of Translation», Babel: International JOurnal of Translation, 1985, 31 (2), 77-85.

LeUVEN-ZWART, K. VAN (1989-1990). «Translation and Original: Similarities and Dissimilarities», Target, International Journal of Translation Studies, 1:2, 151181 y 2:1, 69-95.

LORSCHER (1989). «Models of the Translation Process: Claim and Reality», Target, $1(2), 43-68$.

Martin Mingorance, L. (1984). «Lexical Fields and Stepwise Lexical Decomposition in a Contrastive English-Spanish Verb Valency Dictionary», en Hartmann R.R.K. (ed.) Lexeter'83 Proceedings, Tubingen: M. Niemeyer, 226-236.

MARTIN Mingorance, L. (1985). «La semántica sintagmática del adjetivo: parámetros para la organización de un lexicón inglés/espanol de valencias adjetivales», Actas del II Congreso Nacional de Lingüística Aplicada. Madrid: AESLASGEL, pp. 329-340.

Martin Mingorance, L. (1987). «Semes, Semantic Classemes, and Dimensions: The Lexicological and Lexicographic Perspectives». Paper read at the XIVth International Congress of Linguists. Berlin, 10-15. August, 1987.

MARTtN Mingorance, L. (1990). «Functional Grammar and Lexematics in Lexicography», en J. Tomaszczyck y B. Lewandowska-Tomaszczyck (eds.) Mecaning and Lexicography, Amsterdam: John Benjamins, 227-253.

MARTIN MingoranCE, L. (1994). «Predicate Schemata and Cognitive Schemata in Speech Production and Interpretation. Towards an Integration of ICMs in an FG Lexicon». Conferencia pronunciada en el VI Congreso Internacional de Gramática Funcional celebrado en la Universidad de Yok, agosto de 1994.

MALONE, JoSEPH L. (1988). The Science of Linguistics in the Art of Translation, Albany, N.Y.: Suny Press.

Marmaridou, A.S.S. (1987). «Semantic and Pragmatic Parameters of Meaning: On the Interface between Contrastive Text Analysis and the Production of Translated Texts», Journal of Pragmatics: An Interdisciplinary Bi-monthly of Language Studies, 11(6), 721-736.

HewsON, L. y MARTIN, J. (1991). Redefining Translation: The Variational Approach, Londres y N. York: Routledge.

NeUBert, A. (1985). Text and Translation, Leipzig: Verlag Enzyklopaedie.

NeUBERT, A. (1986). «Translatorische Relativitaet», en M. Snell-Hornby (ed.), Ubersetzungswissenchaft-Eine Neuorientierung, Tubingen: Francke Verlag, 85-105. Newmark, P. (1981). Approaches of Translation, Oxford: Pergamon Press. 
NeWMark, P. (1988). A Textbook of Translation, New York: Prentice Hall.

NeWmark, P. (1991). About Translation, Clevendon: Multilingual Matters.

Newmar, P. (1992). Manual de traducción, (trad. esp., Virgilio Moya) Madrid: Cátedra.

NidA, E. (1985). «Translating Means Translating Meaning. A Sociosemiotic Approach to Translating», Xth World Congress of F.I.T. (119-125).

OlBERTZ, HELLA (1987). «Enredos sintácticos en la comparación de traducción y original. Algunas sugerencias de cómo solucionarlos dentro del modelo comparativo de Kitty van Leuven-Zwart», Amsterdam: Dpto. de Estudios Hispánicos, Universidad de Amsterdam (Tesina doctoral de Vertaalwetenschap).

PETOFI, J.S. (1984). «Funciones de expresion, oraciones, actos comunicativos, textos: aspectos del significado y de su tematización en la estructura de una teorfa textual» (trad. esp., Tomás Albaladejo), Estudios de Linguíística, 2, 207-231.

Popovic, A. (1970). «The Concep «Shift of Expression» in Translation Analysis», en J. Holmes (1970), The Nature of Translation, The Hague: Mouton, 78-87.

ProchazKA, V. (1964). «Notes on Translating Technique», en P.L. Garvin (ed.) A

Prague School Reader on Esthetics, Literary Structure, and Style, Washington

D.C.: Georgetown University Press, 93-112.

RABADAN, R. (1989). Equivalencia translémica y traducción inglés-español, León: Universidad de León (Tesis Doctoral).

Ross, S.D. (1981). *Translation and Similarity* en M.G. Rose (ed.), Translation Spectrum. Essays in Theory and Practice, Albany: Suny Press, 8-22.

SANCHEZ, J.M. (1993). «The Lexicologist's Reach in the Determination of Macrostructural Patterns in Literary Translation: Justine's love», en Jorge FernandezBarrientos Martín (ed.), Actas de las I Jornadas Internacionales de Lingüística Aplicada (vol. II), I.C.E.: Granada, 293-303.

SANCHEZ, J.M. (1995b). «Una estructuración del campo léxico-conceptual del amor previa a su estudio traductologico inglés-español en The Alexandria Quartet», Atlantis (en prensa).

SÁNCHEZ, J.M. (1995c). «Fundamentos para una metodología descriptiva en el estudio traductológico del texto narrativo: I. Puntos de partidaw, Translatio, XV (en prensa).

SÁNCHEZ, J.M. (1995d). «Fundamentos para una metodología descriptiva en el estudio traductológico del texto narrativo: II. Condiciones de aplicación». Translatio, XV (en prensa).

SÁNCHEZ, J.M. (1995e). «Desplazamientos léxico-semánticos y efectos macroestructurales en la traducción española de The Alexandria Quartet: topología conceptual», Miscelanea. A Journal o English and American Studies (Zaragoza), 16, 189-213.

SCHULTE, R. (1979). «The Act of Translation: From Interpretacion to Interdisciplinary Thinking», Translation Review, 4, 3-8.

STEINER, G. (1975). After Babel: Aspects of Language and Translation, Londres: Oxford Press. 
TOURY, G. (1978). «The Nature and Role of Norms in Literary Translation», en J.S. Holmes et al. (eds.) (1978), Literature and Translation: New Perspectives in Literary Studies, Leuven: Acco, 83-100.

Toury, G. (1980a). «The Adequate Translation as an Intermediating Construct: A Model for the Comparison of a Literary Text and Its Translation» en G. Toury, (1980), In Search of a Theory of Translation, Tel Aviv: Porter Institute, 112121.

TouRY, G. (1980b). «Toward Descriptive Translation Studies: Goals, Procedures, and Some Basic Notionsw en G. Toury, (1980), In Search of a Theory of Translation, Tel Aviv: Porter Institute, 79-89.

TourY, G. (1985). «A Rationale for Descriptive Translation Studies», en T. Hermans (ed.) The Manipulation of Literature: Studies in Literary Translation, Londres: Croom Helm, 16-41.

TourY, G. (1986). «Monitoring Discourse Transfer: A Test-Case for a Developmental Model of Translationw, en J. House y Shoshana Blum-kulka (eds.) (1986), Interlingual and Intercultural Communication, Tuebingen: G. Narr, 79-94.

TOURY, G. (1989). «Well, What about a Linguistic Theory of Literary Translation?», Bulletin de la Commission Interuniversitaire Suisse de Linguistique Apliquee, 49, 102-105.

VOORT, C. VAN DER (1991). «Narratology and Translation Studies», en K. van Leuven-Zwart y T. Naaijkens (eds.), Translation Studies: The State of the Art, Proceedings of the First James S. Holmes Symposium on Translation Studies, Amsterdam: Rodopi, 65-73.

Welt Trahan, E. (1980). *The Translator as Involuntary Critic», Pacific Quarterly Moana, 5(1) (núm. esp. de traducción), 33-38. 